\title{
Validation of DLR's sound shielding prediction tool using a novel sound source
}

\author{
Karl-Stéphane Rossignol*, Markus Lummer ${ }^{\dagger}$, Jan Delfs ${ }^{\ddagger}$ \\ German Aerospace Center (DLR), Lilienthalplatz 7, D-38108 Braunschweig
}

This paper is concerned with an experimental validation methodology for DLR's acoustic shielding prediction boundary element code BEMPAR. This code in turn will be integrated into the overall aircraft noise prediction tool PANAM of DLR. The presented validation concept is based on a novel laser-based sound source. Almost perfect monopole-type test signals may be produced with a frequency content up to roughly $100 \mathrm{kHz}$ in combination with a very small source extension. These characteristics make this technique especially attractive for shielding/installation tests, which typically have to be performed at relativey small scale. BEMPAR is a boundary element code (BEM) which solves for the scattered pressure field. Three generic test cases are evaluated, a circular plate, a long cylinder and DLR's low noise aircraft (LNA-1) nacelle model. The outcome of this investigation clearly demonstrate the potential of BEMPAR for the prediction of installation effects.

\section{Nomenclature}

$\begin{array}{ll}D & \text { Diameter } \\ O A S P L & \text { Overall Sound Pressure Level } \\ \gamma & \text { Shielding factor } \\ \langle\rangle & \text { Ensemble average } \\ \phi & \text { Azimuthal angle } \\ \theta & \text { Polar angle } \\ \vartheta^{\prime} & \text { Temperature fluctuations } \\ f & \text { Frequency } \\ p^{\prime} & \text { Pressure fluctuations } \\ \boldsymbol{x} & \text { Field point } \\ \boldsymbol{y} & \text { Point on body surface } \\ G & \text { Green's function of the Helmholtz equation } \\ L & \text { Integral operator in the Kirchhoff equation } \\ M & \text { Integral operator in the Kirchhoff equation } \\ M^{t} & \text { Integral operator in the Burton/Miller equation } \\ N & \text { Integral operator in the Burton/Miller equation } \\ p_{I} & \text { Incident pressure fluctuation } \\ p_{S} & \text { Scattered pressure fluctuation } \\ p_{T} & \text { Total pressure fluctuation }\end{array}$

\section{Introduction}

BEAUSE of the actual awareness regarding transport aircraft noise and current or future noise regulations, research is being conducted for the development of future generation low-noise airplanes.

\footnotetext{
${ }^{*}$ Research Engineer, Department of Technical Acoustics, Institute of Aerodynamics and Flow Technology, German Aerospace Center (DLR)

${ }^{\dagger}$ Research Engineer, Department of Technical Acoustics, Institute of Aerodynamics and Flow Technology, German Aerospace Center (DLR)

${ }^{\ddagger}$ Head of the department of Technical Acoustics, Institute of Aerodynamics and Flow Technology, German Aerospace Center (DLR)
} 
To meet the requirements associated with such aircraft, noise prediction tools are being developed which can be integrated into the design phase at an early stage.

Aircraft noise may be subdivided into two categories; airframe noise and propulsion noise. Airframe noise is mainly influenced by the geometry of the aircraft components (e.g. high lift devices, landing gears) while propulsion noise refers to sound produced in the turbomachinery, jet or propellers. Noise from both categories in addition depends on the actual operational conditions (e.g. flight speed, wing settings, etc.). Any sucessful reduction of air traffic noise necessitates a lowered contribution of all these noise sources simultaneaously. Moreover, interaction among them, or installation effects, also needs to be taken into account to accurately predict the noise characteristics of an aircraft or the noise reduction potential of an aircraft design.

During the last few years DLR has developed a global airframe noise prediction tool (PROFAN) based on the semi-empirical modelling of individual noise sources. ${ }^{1}$ The overall aircraft noise prediction tool PANAM, ${ }^{2}$ integrates PROFAN as well as simple jet ${ }^{3}$ and fan $^{4}$ noise prediction schemes to study the influence of different flight trajectories and aircraft configurations on each individual noise source. Moreover, fly-over noise foot prints can be monitored. Because of the semi-empirical nature of its component source models, PANAM is not computationally intensive and can be easily applied in a design optimization chain.

In its present state, the code does not however account for installation effects. In an effort to close this gap, sound shielding prediction tools have been developed at DLR taking into account wave propagation as well as wave diffraction effects. These methods (BEM code BEMPAR and raytracing code SHADOW) have the potential of enabling the prediction of the sound shielding properties of airplane components of arbitrary geometry and therefore installation effects.

The shielding prediction codes need experimental validation. To proceed, a recently developed reference sound source was used to simulate engine noise in a non-intrusive manner using a laser. ${ }^{5}$ The objective of the present study is to validate DLR's boundary element solver (BEMPAR) for sound shielding prediction using this new sound source. Simple shielding experiments on generic geometries were performed and compared to numerical calculations. Tests were performed without the influence of a mean flow field as in the current simulations. Three tests cases were defined, beginning with two very simple geometries namely a circular flat plate and a long cylinder. Finally, the shielding effect of an engine nacelle model was evaluated. The presented results constitute a first step towards a detailled validation of our procedure.

\section{The Boundary Element Method (BEM)}

The DLR sound shielding prediction tool BEMPAR is a boundary element code which solves the exterior Helmholtz problem (scattering problem). It is a BEM method for triangulated surfaces based on the algorithm of S.Kirkup. ${ }^{6}$

\section{A. The Burton/Miller Equation}

To solve a scattering problem with BEM the pressure field $p_{T}(\boldsymbol{x})$ is split into a given incident field $p_{I}(\boldsymbol{x})$ and a scattered field $p_{S}(\boldsymbol{x})$ :

$$
p_{T}(\boldsymbol{x})=p_{I}(\boldsymbol{x})+p_{S}(\boldsymbol{x}) .
$$

The scattered field has to be determined such that at the boundary $\partial A$ of the body, the normal derivative of the total pressure $p_{T}$ vanishes, i.e., one has

$$
\frac{\partial p_{S}}{\partial n}=-\frac{\partial p_{I}}{\partial n} .
$$

For the determination of the scattered pressure one uses Kirchhoff's formula written for the exterior domain

$$
M p_{S}-c p_{S}(\boldsymbol{x})=L \frac{\partial p_{S}}{\partial n_{y}}
$$

where $L$ and $M$ are the integral operators

$$
L \frac{\partial p_{S}}{\partial n_{y}}=\oint_{\partial A} G(\boldsymbol{x}-\boldsymbol{y}) \frac{\partial p_{S}(\boldsymbol{y})}{\partial n_{y}} d A, \quad M p_{S}=\oint_{\partial A} p_{S}(\boldsymbol{y}) \frac{\partial G(\boldsymbol{x}-\boldsymbol{y})}{\partial n_{y}} d A .
$$


$G$ is the Green's function of the Helmholtz equation

$$
G(\boldsymbol{x}-\boldsymbol{y})=\frac{e^{i k r}}{4 \pi r}, \quad r=|\boldsymbol{x}-\boldsymbol{y}|=\sqrt{\left(x_{k}-y_{k}\right)^{2}},
$$

$d A$ is an element of the body surface, and $c$ a constant that takes the following values

$$
c=\left\{\begin{array}{ll}
1 & , \text { point } \boldsymbol{x} \text { inside of the domain } \\
\frac{1}{2} & , \text { point } \boldsymbol{x} \text { on the boundary } \\
0 & , \text { point } \boldsymbol{x} \text { inside of the body }
\end{array} .\right.
$$

The normal derivative appearing on the right-hand side of Eq. (3) is known from Eq. (2). Thus, if $\boldsymbol{x}$ is a point on the boundary of the body, we obtain the following integral equation for the determination of $p_{S}(\boldsymbol{x})$ on the surface

$$
M p_{S}-\frac{1}{2} p_{S}=-L \frac{\partial p_{I}}{\partial n_{y}}
$$

It is well known that the Kirchhoff approach suffers from numerical artefacts ${ }^{7,8}$ which stem from the coupling of the exterior and interior boundary value problems. If the associated interior Dirichlet problem has an eigenvalue at the considered wave number, the solution of the exterior problem becomes undetermined. Some workarounds are known. One of them, which is used in BEMPAR, is the so-called Burton $/$ Miller $^{9}$ approach that decouples the interior and exterior problem but requires the approximation of more singular integrals.

In order to derive the Burton/Miller equation one differentiates the Kirchhoff equation Eq. (3) with respect to the normal direction at the point $\boldsymbol{x}$ and gets

$$
\frac{\partial}{\partial n_{x}} M p_{S}-\frac{1}{2} \frac{\partial p_{S}}{\partial n_{x}}=\frac{\partial}{\partial n_{x}} L \frac{\partial p_{S}}{\partial n_{y}}
$$

The multiplication of this equation with a complex constant $\beta$ and addition to the Kirchhoff equation yields the Burton/Miller equation ${ }^{9}$

$$
M p_{S}-\frac{1}{2} p_{S}+\beta N p_{S}=L \frac{\partial p_{S}}{\partial n_{y}}+\beta M^{t} \frac{\partial p_{S}}{\partial n_{y}}+\frac{\beta}{2} \frac{\partial p_{S}}{\partial n_{x}}
$$

where $M^{t}$ and $N$ are the integral operators

$$
M^{t} \frac{\partial p_{S}}{\partial n_{y}}=\frac{\partial}{\partial n_{x}} L \frac{\partial p_{S}}{\partial n_{y}}=\oint_{\partial A} \frac{\partial G(\boldsymbol{x}-\boldsymbol{y})}{\partial n_{x}} \frac{\partial p_{S}(\boldsymbol{y})}{\partial n_{y}} d A, \quad N p_{S}=\frac{\partial}{\partial n_{x}} M p_{S}=\oint_{\partial A} p_{S}(\boldsymbol{y}) \frac{\partial^{2} G(\boldsymbol{x}-\boldsymbol{y})}{\partial n_{x} \partial n_{y}} d A
$$

The complex constant $\beta$ has been set to $\beta=i / k$.

In order to solve the Burton/Miller equation (9) numerically, in BEMPAR the method of S. Kirkup ${ }^{6}$ is used which discretizes the integral operators for plane triangles and constant source strengths. Then equation (9) becomes a system of linear equations for the unknown surface pressure values. The system's matrix is complex, fully occupied and asymmetrical. The Fortran subroutines of S.Kirkup ${ }^{\mathrm{a}}$ have been translated to $\mathrm{C}++$ and the linear system is solved using the parallel linear algebra routines from the ScaLAPACK software package ${ }^{\mathrm{b}}$.

If the surface pressure $p_{S}(\boldsymbol{y})$ is known, the scattered pressure in the domain of the solution can be calculated from the Kirchhoff equation Eq. (3), setting $c=1$.

A surface triangulation of simple bodies can be obtained by the grid generation program netgen ${ }^{c}$, which is made for the generation of volume grids for FEM calculations but also provides the possibility to create STL-files ${ }^{\mathrm{d}}$ of the body surface.

\footnotetext{
${ }^{a}$ http://http://www.boundary-element-method.com

bhttp://www.netlib.org

${ }^{\mathrm{c}}$ http://www.hpfem.jku.at/netgen

d STL stands for Stereolithography and the STL-format is a simple surface description using triangles, which is often used in rapid prototyping.
} 


\section{Experimental Validation}

\section{A. Principle of Laser Generated Sound}

By focussing a high energy laser it is possible to locally heat a very small volume of air up to ionisation. This process than leeds to the creation of a small plasma zone which rapidly expands in all directions. ${ }^{10}$ The expanding plasma in turn generates an omni-directional pressure wave with initially supersonic propagation speed. From the wave equation for the pressure perturbation $p^{\prime}$ in a stagnant medium of variable mean density $\rho^{0}(x)$ the above phenomenon can be described in the following way,

$$
p^{\prime}(\mathbf{x}, t)=\left.\frac{1}{4 \pi r} \frac{1}{c_{p} T^{0}} \frac{\delta \dot{\vartheta}^{\prime}}{\delta t}\right|_{\tau}
$$

where $r$ is the distance from the source, $\tau$ the retarded time, $c_{p}$ the specific heat capacity at constant pressure and $T^{0}$ the absolute temperature in the surrounding environment. This relation emphasize the importance of the temporal heat input $\left(\frac{\delta \dot{\vartheta}^{\prime}}{\delta t}\right)$ in generating a high amplitude pressure wave. Moreover such a pressure wave does not exhibit any directionality and decreases linearly away from its origin. The generated shock wave slows down to the speed of sound after approximately $20 \mu$ s at which point it propagates as an isentropic acoustic wave. Consequently the small plasma generated can be seen as a breathing sphere with 10 mm radius. ${ }^{11}$

For our purpose, a conventional Gemini double cavity PIV laser from New Wave Research was mounted on the setup shown in figure 1. This laser is capable of producing a stable mean output power of $120 \mathrm{~mJ}$ with a pulse width of $3-5 \mathrm{~ns}$ at a repetition rate of up to $15 \mathrm{~Hz}$. The laser (\#1) is coupled to a small mouting traverse (\#4) which supports the diverging optic (\#2) and the converging lens (\#3). The whole setup rests on a support beam ensuring a secure fixation of all parts. In order to confine a maximal amount of energy on the focal point the ouput beam of the laser is enlarged to four time its initial diameter by means of a low-loss diverging optic. Afterwards, the beam is focused by a $50 \mathrm{~mm}$ focal length lens.

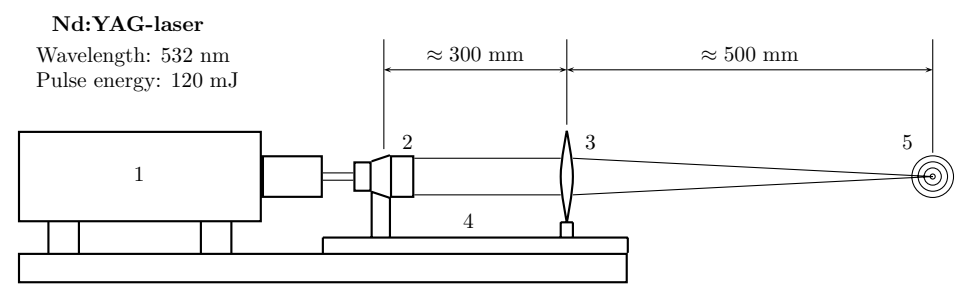

Figure 1: Sketch of the laser setup used to generate a pressure pulse. \#1, laser; \#2, broadening optic; \#3, converging lens; \#4, mounting traverse; \#5, plasma spot.

Laser-based sound generation enables the production of a nearly omni-directionnal broadband pressure wave in a non-intrusive manner. ${ }^{5,11}$ This pressure wave can reach a mean peak sound pressure level (SPL) of up to $90 \mathrm{~dB}$ at $60 \mathrm{kHz}(O A S P L=119 \pm 1 \mathrm{~dB}) \cdot{ }^{11}$ It also exhibits a repeatable spectral distribution with a standard deviation less than $1.9 \mathrm{~dB}$ over the range $0-80 \mathrm{kHz}$.

\section{B. Data Acquisition and Post-Processing}

Since we are dealing with a pulse of very short duration $(\approx 50 \mu \mathrm{s})$, very high data acquisition rates are needed. The available acquisition unit (GMB Viper, 48 channel) can perform measurements at a maximal acquisition rate of $250 \mathrm{kHz}$ which theoreticaly enables us to correctly reconstruct signals with frequencies up to approximately $125 \mathrm{kHz}$. In order to measure such frequencies, $1 / 8$ inch G.R.A.S. 40 DP microphones with a $140 \mathrm{kHz}$ dynamic range were used. For the present experiment a laser repetition rate of $10 \mathrm{~Hz}$ and an acquisition time of $20 \mathrm{~s}$ were chosen. This means that 200 pulses are recorded per data point.

Pressure measurements, for each configuration, are performed with a continuously running laser operated at full power, in order to get the maximal possible signal to noise ratio (SNR). The laser output trigger signal is simultaneously registered in order to facilitate data post-processing. For each configuration all pulses are extracted from the measured time series in a two step procedure. First a coarse extraction is done by thresholding based on the laser trigger output. In the second step, a reference signal is selected in order to perform a finer extraction based on its correlation with the raw data. The whole procedure ensures an optimal search for pulses and provides an efficient rejection of spurious pressure pulses. 


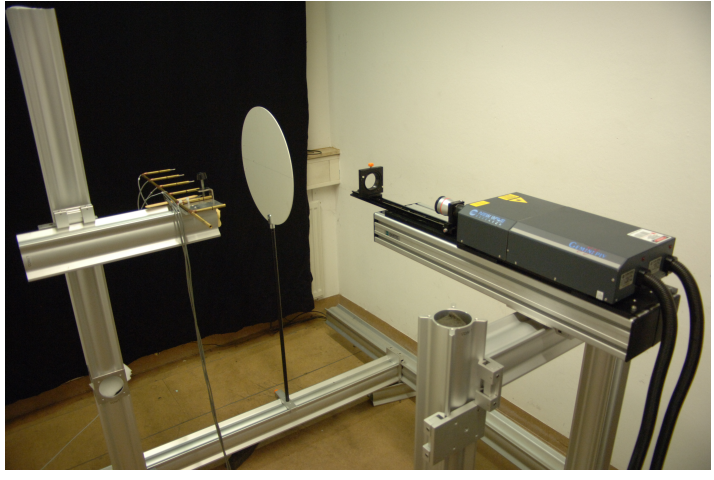

(a) Experimental setup.

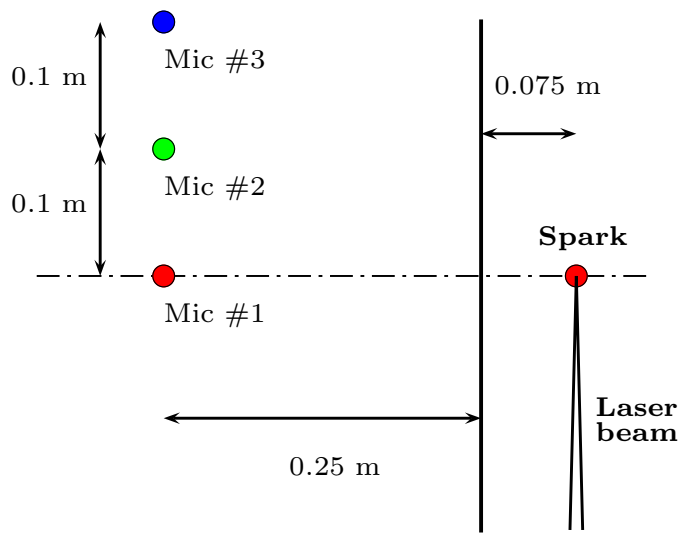

(b) Schematic disposition of all elements as seen from the top. (Not to scale)

Figure 2: Circular plate test case

\section{Test Case 1: Circular Flat Plate}

The first test setup is shown in figure 2a and consists of a basic circular flat plate. It has a diameter of $40.2 \mathrm{~cm}$ and is $3 \mathrm{~mm}$ thick. The plate is held from the bottom by a metal rod of $1.2 \mathrm{~cm}$ in diameter and $80 \mathrm{~cm}$ long. Microphones are placed at a distance of $25 \mathrm{~cm}$ relative to the center of the plate and are $10 \mathrm{~cm}$ appart, the first one being in front of the plate center (see figure $2 \mathrm{~b}$ ). The laser beam comes perpendicularly to the microphones axes and its focus point is aligned with the plate center, $7.5 \mathrm{~cm}$ away from it (32.5 $\mathrm{cm}$ from the first microphone tip).

\section{Test Case 2: Long Cylinder}

The cylinder setup is shown in figure $3 \mathrm{~b}$. As for the circular plate, it is held from the bottom, through its axis, by a metal rod of $2 \mathrm{~cm}$ diameter and $59.5 \mathrm{~cm}$ long. The cylinder itself has a diameter of $8 \mathrm{~cm}$ and is $80 \mathrm{~cm}$ long. For this test case, the same microphone support as before is used but with a different microphone alignment. The second microphone is aligned with the geometrical center of the cylinder and both microphones 1 and 3 are $10 \mathrm{~cm}$ appart from the central one (see figure 3a). The laser spark is generated $11.5 \mathrm{~cm}$ away from the cylinder geometrical center and is $40.5 \mathrm{~cm}$ away from the second microphone.

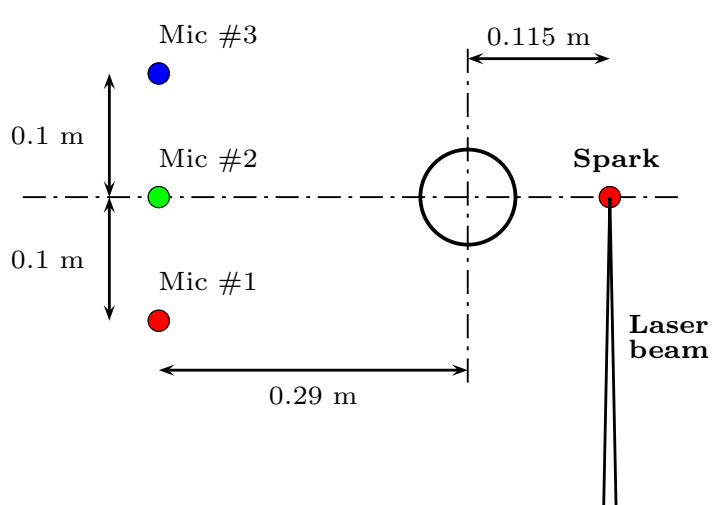

(a) Schematic disposition of all elements as seen from the top. (Not to scale)

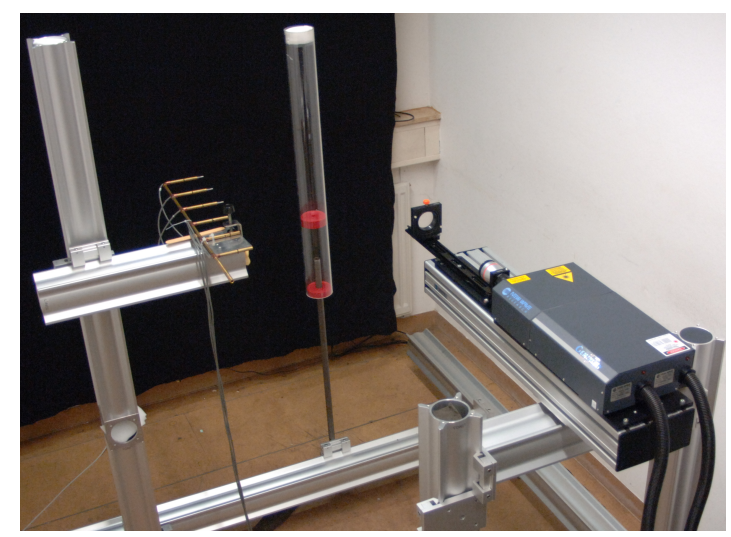

(b) Experimental setup for the cylinder test case.

Figure 3: Long cylinder test case.

\section{E. Test Case 3: LNA-1 Nacelle Model}

It was already shown that a laser-based sound source can experimentally be used to evaluate the effect of overwing engine position on "fly-over" noise contours. ${ }^{12}$ These preliminary studies where however performed without taking into account diffraction effects caused by the engine nacelle itself. The laser 
sound source position was simply varied and the corresponding shielding factor maps underneath the LNA-1 model where measured. It seems logical to assume that the engine nacelle geometry will act on the nearly omni-directional SPL of the laser sound source to modify its directivity and therefore modulate the measured shielding factor maps. That information is however not known and needs to be experimentally acquired in order to accurately evaluate the effect of engine position. It is necessary here to emphasize that the present experiment cannot be compared to reality. One would have to generate a sound source having the actual directivity of an airplane turbine. This third test case is essentially a generic way of validating our computational code BEMPAR.

In order to further evaluate the capacity of the noise shielding prediction scheme we used DLR's LNA-1 nacelle model (see figure 4 and 5). It is an asymmetrical model, as it can be seen in the section cuts of figures $4 \mathrm{a}$ and $4 \mathrm{~b}$, having a thinner upper surface than its bottom one. The model is $19 \mathrm{~cm}$ long and $9 \mathrm{~cm}$ wide at the nozzle exit (right side). Engine noise is simulated by the laser sound source, 9.5 $\mathrm{cm}$ from the nacelle front on its central axis, at the origin of the coordinate system of figures $4 \mathrm{a}$ and $4 \mathrm{~b}$ $(x=0, y=0, z=0)$. The pressure field around the model is measured using one $1 / 8$ inch G.R.A.S. 40 DP microphone which is attached to a rotating ring covered with foam (see figure 5). The microphone is located $22 \mathrm{~cm}$ away from the plasma spot. For this work, one polar angle $\left(\theta=0^{\circ}\right)$ and six azimuthal angles $\left(\phi=12^{\circ}, 17^{\circ}, 22^{\circ}, 32^{\circ}, 42^{\circ}, 52^{\circ}\right)$ are considered.

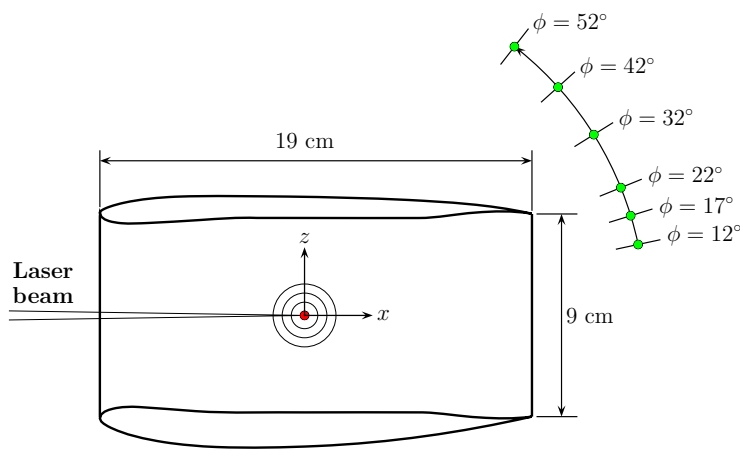

(a) Top view

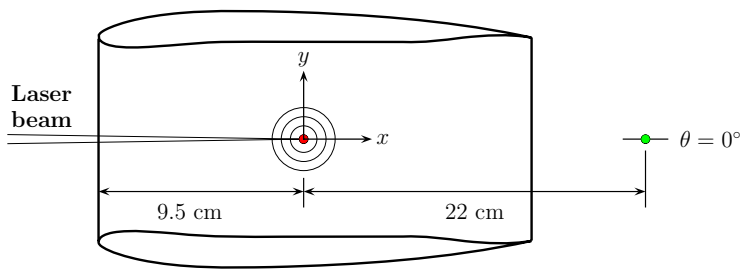

(b) Side view

Figure 4: Dimensions of the LNA-1 nacelle model and selected measurement positions.

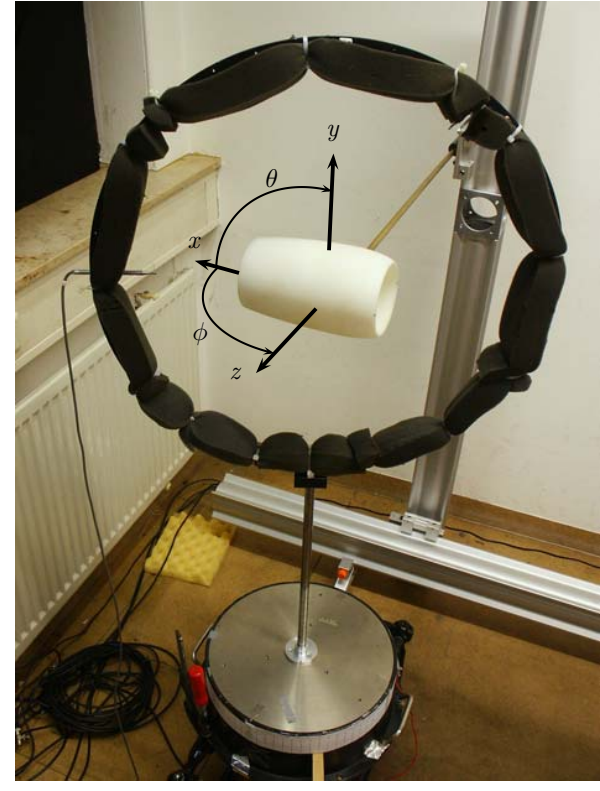

Figure 5: Experimental setup for the LNA-1 nacelle test case. The laser beam is coming from the right-hand side of the picture.

\section{Results}

\section{A. Anechoic versus Non-Anechoic Conditions}

Because of the very short duration of the plasma spark, it was realized that a low reflection environment is not absolutely necessary for our purpose. Reflections from any part of the mounting setup or off the room's walls can easily be identified as damped pulses in the acquired raw signals and rejected. They are also much weaker, with amplitudes about 10 times lower as the actual pulse of interest. This means that one is able to correctly recover spectral information from the generated pressure pulses through careful data-processing, even in hard-walled rooms.

This hypothesis was studied in both an anechoic room and a hard-wall room (without any sound absorption modification). A comparison of measured spectra in both environments is presented in figure 6. From these results, it is clear that in the range from 5 to $25 \mathrm{kHz}$ there is no notable influence on the laser spark SPL spectrum, with differences on the order of less than $1 \mathrm{~dB}$ over the whole frequency range.

Because of the above mentioned observations, all results that are presented in this paper were obtained 


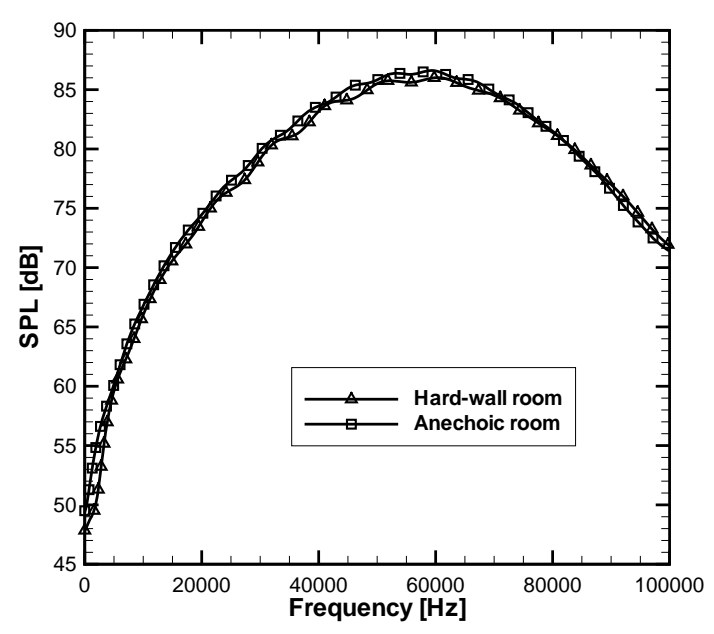

(a) Range $0-100 \mathrm{kHz}$

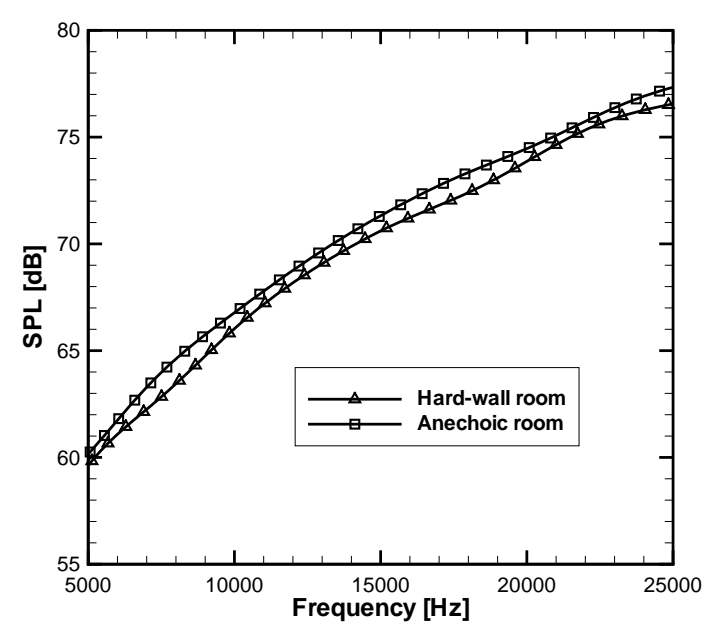

(b) Range $5-25 \mathrm{kHz}$

Figure 6: Comparison of measured SPL in an anechoic room versus measurements in a hard-walled room $(\Delta f=61 \mathrm{~Hz})$. Measurements of the isolated laser sound source pressure pulse without shielding element at $90^{\circ}$ relative to the laser beam incidence and $\approx 40 \mathrm{~cm}$ away from the laser focus point (sound source origin).

in a hard-walled room. All parts of the setup were disposed in such a way as to minimize possible interferences between acoustic reflections and the laser generated pressure pulse. Moreover, lots of efforts have been made in order to align all components with the greatest possible precision and great care was taken to ensure an optimal spark generation. These last parameters are identified as deciding for the achievement of repeatable results and are much more important than the level of background noise or reflections.

\section{B. Sound Shielding by a Circular Plate and a Long Cylinder}

The shielding factor is defined in the following way,

$$
\gamma(f)=\frac{\left\langle p_{T}(f)\right\rangle}{\left\langle p_{I}(f)\right\rangle}
$$

where $\left\langle p_{T}(f)=p_{S}(f)+p_{I}(f)\right\rangle$ and $\left\langle p_{I}(f)\right\rangle$ are the ensemble averaged total and incident pressure fluctuations. Both measurements are performed sequentially without modifications to the setup. First, measurements with the plate are done, it is then removed and the unshielded pressure field is acquired.

A comparison of the computed (BEM) and measured shielding factors in the range 5 to $25 \mathrm{kHz}$ for the circular plate case is shown in figure 7a. A very good agreement is found for microphone \#2 with variations on the order of $1 \mathrm{~dB}$ except where attenuation peaks exists. Overall the shape of the shielding factor spectrum is well captured in the considered range. For microphone \#1, directly behind the plate center, we observe a very different spectrum with a peak value of approximately 0.85 at $5 \mathrm{kHz}$ and less fluctuations of the shielding factor. These observations matches well with the theory of wave diffraction by a circular plate which also predict a concentrated region of pressure amplification directly behind the circular plate center point, called Arago spot.

For this microphone position agreement between experiment and computation is found to be not as good as for microphone \#2. It is hypothesized that position uncertainties alone are responsible for these differences. In fact, a second computed shielding factor spectrum for a slightly eccentric position $(r=0.005 \mathrm{~m})$ is also plotted in figure 7a showing a much better agreement between experiment and computation $(\approx 1 \mathrm{~dB})$. This reveals the strong spatial dependency of the shielding factor in the central region behind the plate which was furthermore confirmed from the BEM shielding factor maps (see figure $7 \mathrm{~b})$.

For the cylinder test case, a comparison of the shielding factor for microphone \#1 and \#2 in the range 5 to $25 \mathrm{kHz}$ is plotted in figure 8a. Microphone \#3 is ignored because of the setup symmetry. In the present case the measured shielding factor at microphone $\# 1$ is also seen to agree very well with 


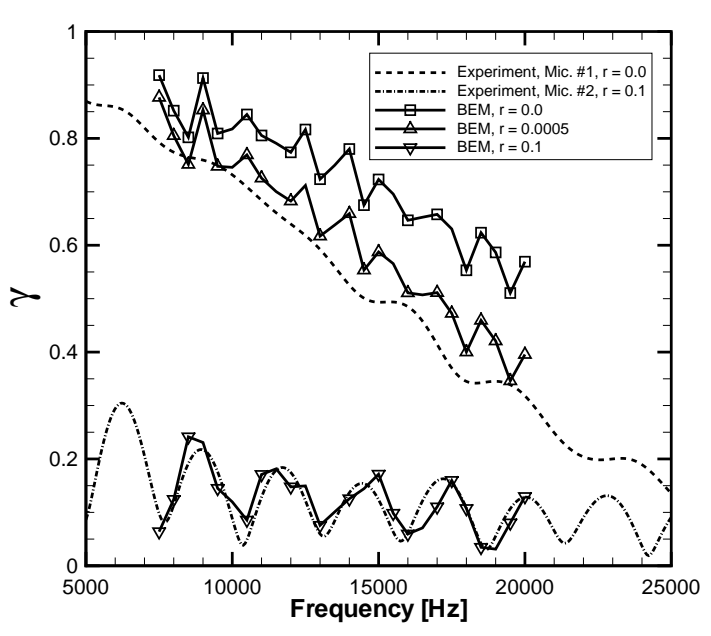

(a) Measured shielding factor spectrum.

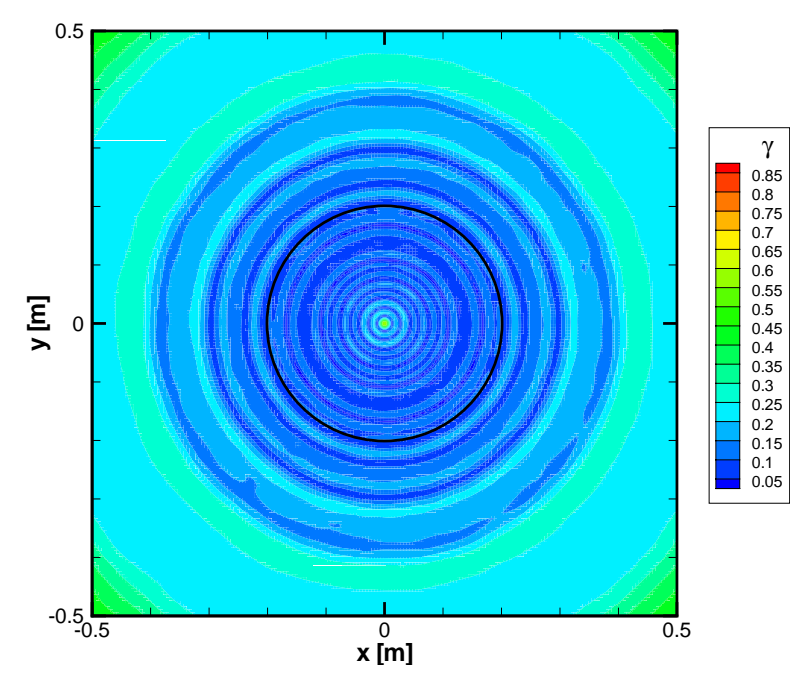

(b) Computed shielding factor map at $f=17 \mathrm{kHz}$. The geometry is shown in black

Figure 7: Comparison between computed and measured shielding factors for the circular plate test case.

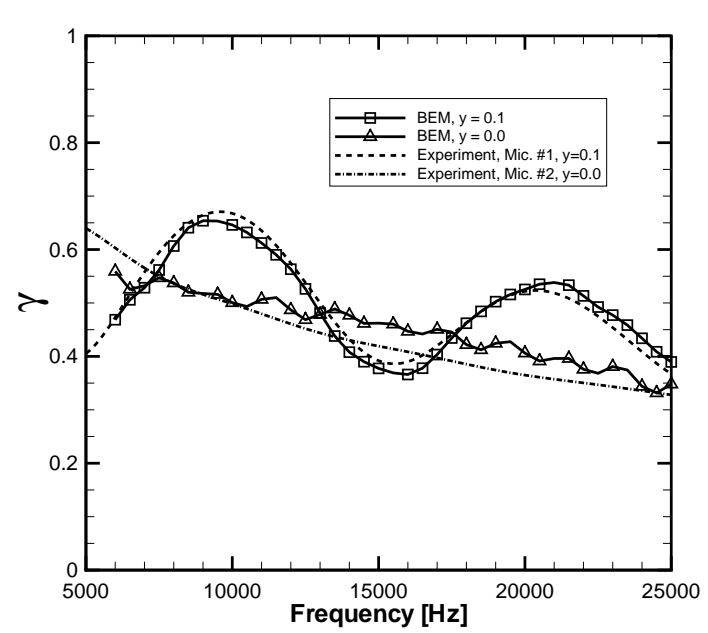

(a) Measured shielding factor spectrum.

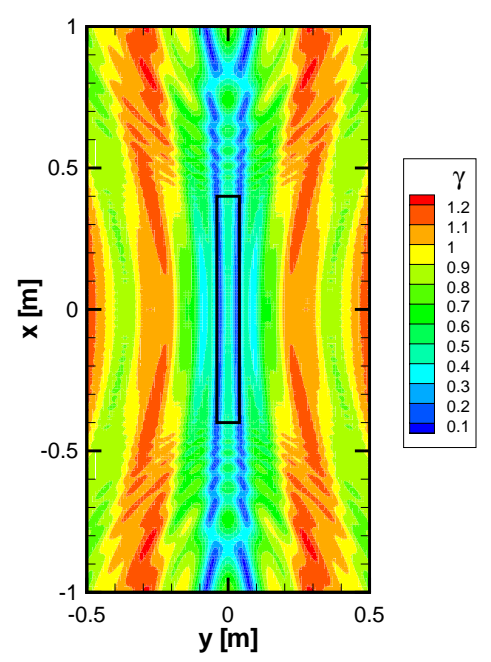

(b) Computed shielding factor map at $f=17 \mathrm{kHz}$. The geometry is shown in black.

Figure 8: Comparison between computed and measured shielding factors for the long cylinder test case.

the BEM computation with overall variations of less than $1 \mathrm{~dB}$. Here remember that microphone \#1 is located $10 \mathrm{~cm}(1.25 \mathrm{D})$ away from the central axis (see section $\mathrm{D}$ ). The comparison at microphone \#2 is not as good but still presents maximum differences of the order of $1 \mathrm{~dB}$ which is comparable to the results presented in figure 7a for the circular plate case. Here it is once again important to emphasize that positioning of the microphone is a critical aspect. A zone of large shielding factor gradients exists behind the cylinder as shown from the BEM computations 2D maps (see figure $8 \mathrm{~b}$ ).

\section{Sound Shielding from the LNA-1 Nacelle Model}

The measured shielding factor spectrum (in the range $5-25 \mathrm{kHz}$ ) along with the corresponding BEM computations are presented in figures $9 \mathrm{a}$ to $9 \mathrm{f}$. 


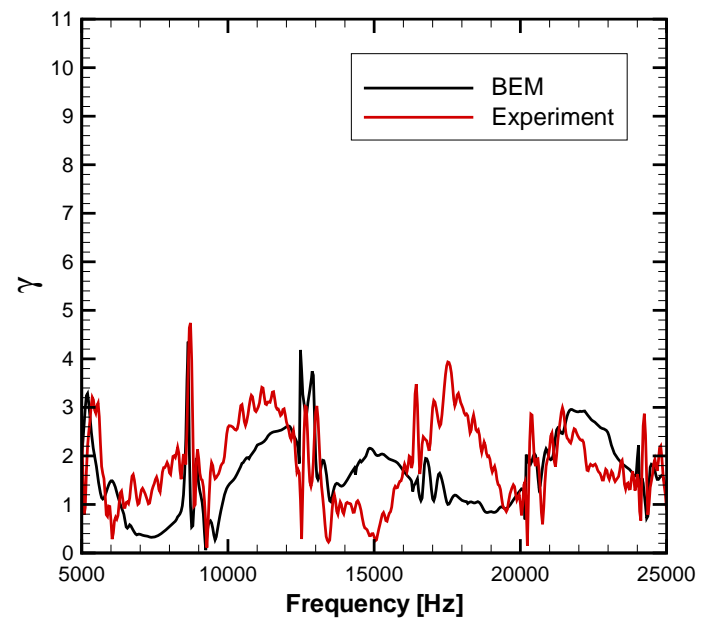

(a) $\theta=0^{\circ}, \phi=12^{\circ}$

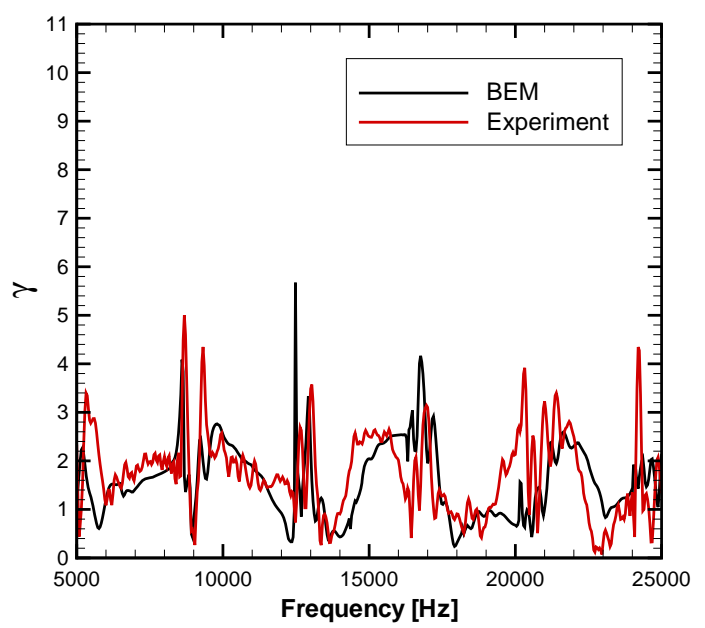

(c) $\theta=0^{\circ}, \phi=22^{\circ}$

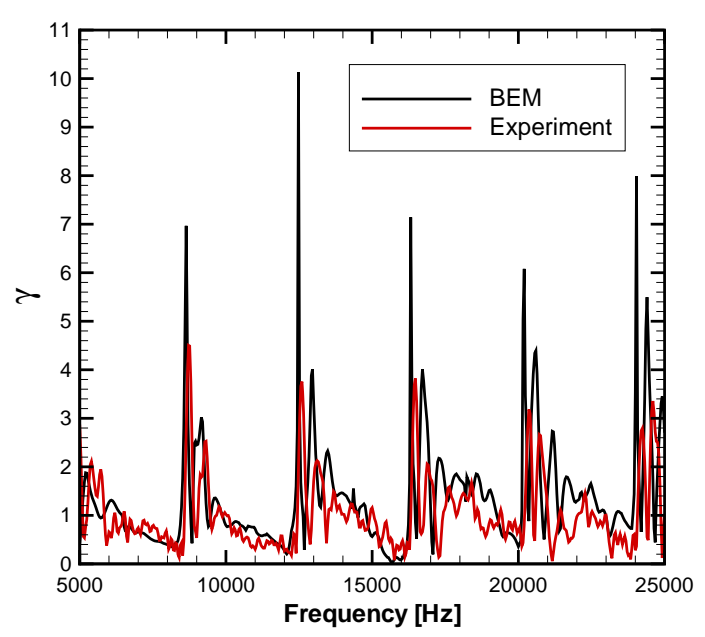

(e) $\theta=0^{\circ}, \phi=42^{\circ}$

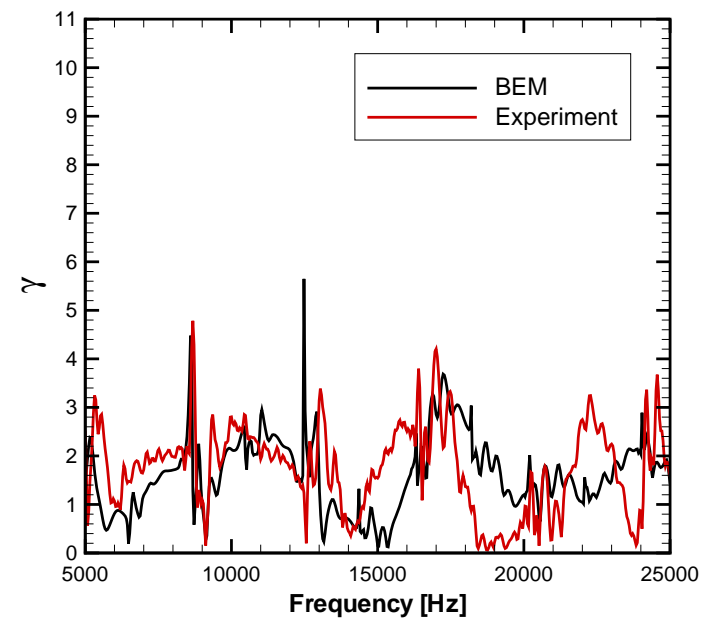

(b) $\theta=0^{\circ}, \phi=17^{\circ}$

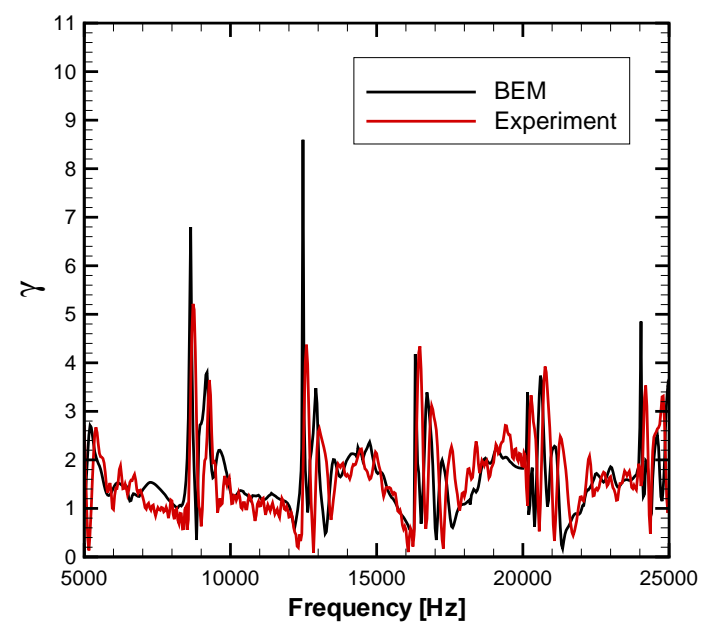

(d) $\theta=0^{\circ}, \phi=32^{\circ}$

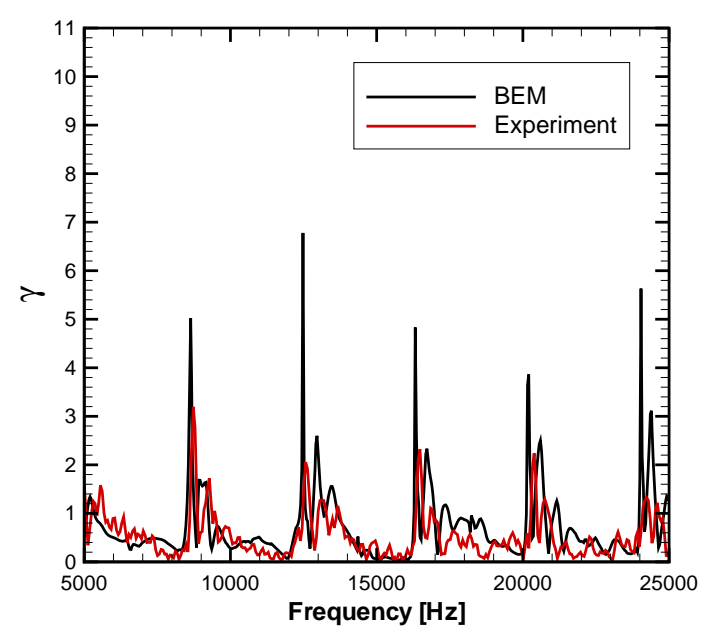

(f) $\theta=0^{\circ}, \phi=52^{\circ}$

Figure 9: Comparison of computed and measured shielding factor for the LNA-1 nacelle model. $(\Delta f=40$ $\mathrm{Hz})$ 
A good overall agreement of the numerical and experimental profiles is observed for measurement angles larger than $17^{\circ}$. The frequencies at which strong amplification or attenuation occurs are always correctly predicted. However, at these angles the corresponding absolute amplification levels do not, most of the time, compare well. Shielding factor differences up to 10 are obtained. Between these peaks, levels tendencies are correctly recovered. At $\phi=12^{\circ}$ and $17^{\circ}$, the quality of the agreement between measurements and computation is not as good. For these three measurement angles, one can clearly observe that the similarity between both spectra worsen as frequency increases. The comparison at $\phi=12^{\circ}$ representing the worst results.

These discrepancies could once again be linked to small position uncertainties in the experiments and the existence of very strong acoustic pressure gradients in the small angle domain $\left(\phi<22^{\circ}\right)$ compared to other angles. Such strong variations may occur at precise frequencies due to constructive interference inside of the model combined to an inherent focussing effect of the Nacelle geometry. Here, BEM calculations (see figure 10) of the shielding factor in the plane $y=0(\theta=0)$ confirm this hypothesis. In the $12.5 \mathrm{kHz}$ case stong gradiens exist nearly everywhere in the computation domain whereas only a concentrated region of pressure variations is observed at $f=15 \mathrm{kHz}$. Consequently, position uncertainties in the measurements will affect shielding factors evaluation in a frequency dependent manner. In regions of high pressure gradients the correct estimation of the shielding factor will be limited by the microphone size as well as its positioning uncertainty.

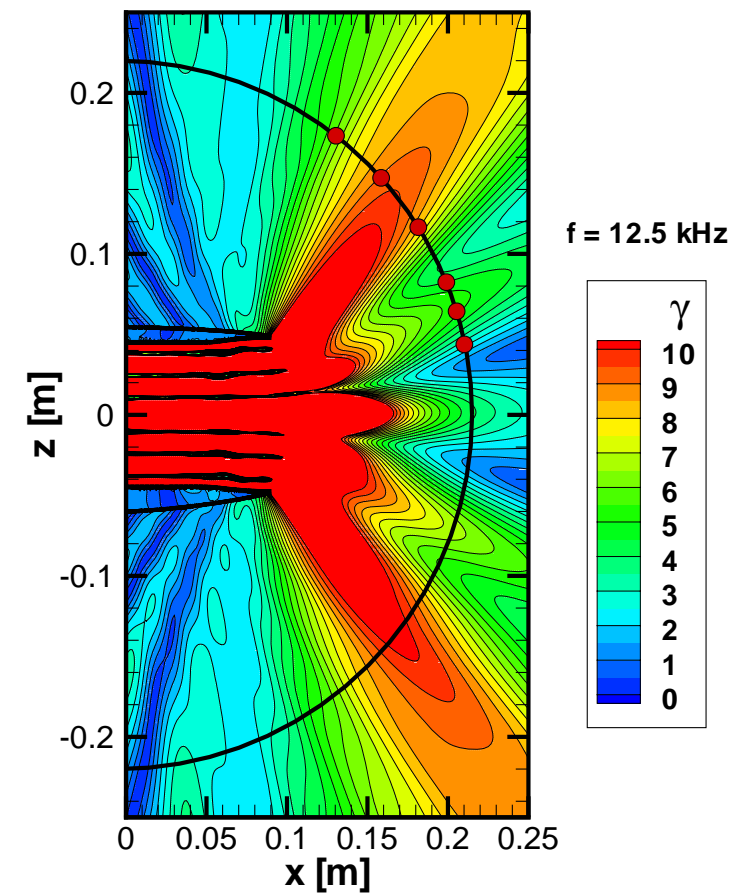

(a) Computed shielding factor map at $f=12.5 \mathrm{kHz}$.

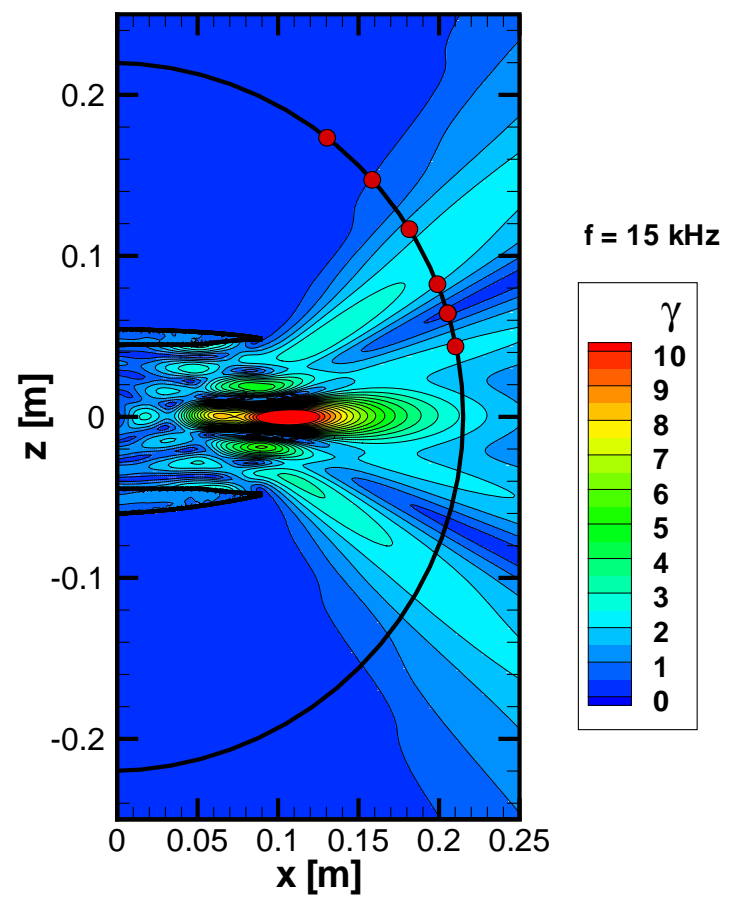

(b) Computed shielding factor map at $f=15 \mathrm{kHz}$.

Figure 10: 2D maps of the shielding factor as computed by the BEM for the LNA-1 nacelle test case. The object geometry is shown in black while the microphone positions are shown by the red dots. The laser beam is coming from the left and the numerical sound source position is at $(x=-0.005, z=0)$

\section{Conlusion}

In order to validate the computational tool for the prediction of shielding effects (BEMPAR) that was developed at DLR, generic shielding experiments using a novel laser-based sound source were done. Three test cases were selected each having an increasing degree of complexity. First, a simple circular flat plate was tested followed by a long cylinder. Finally a nacelle model from DLR's LNA-1 low noise aircraft was chosen.

A very good agreement of the measured and computed shielding factor spectra in the range $5-25$ $\mathrm{kHz}$ was obtained for the circular plate and the long cylinder test cases. Except where attenuation or amplification peaks exist, the BEM prediction is able to recover the measured shielding factor. The tests performed with the LNA-1 nacelle model reveal a much more complicated acoustic spectrum with strong attenuation and amplification peaks. For angles of $\phi=22^{\circ}, 32^{\circ}, 42^{\circ}, 52^{\circ}$ the BEM approach works 
well and correctly predicts the measured shielding factor spectra and their peak frequencies. Absolute shielding factor levels are however constantly overpredicted. Results obtained at $\phi=12^{\circ}, 17^{\circ}$ do not present the same success over the whole spectrum range. These discrepancies between computations and measurements are most probably caused by microphone positioning uncertainties in regions of high acoustic pressure gradients as well as microphone size. In these regions it becomes difficult to perform a validation of the BEMPAR tool because the experimental technique is no longer able to correctly render the complex acoustical pressure field.

The good comparisons obtained in all of the selected test cases still clearly demonstrate the capabilities of DLR's sound shielding prediction tool BEMPAR. They also show that the experimental laser technique is a useful approach, especially due to its ability to generate very high frequency signals non-intrusively. The last characteristic is important for applications in wind tunnels where one wants to have as little impact as possible on the superimposed mean flow.

Furthermore, the knowledge gained during these tests regarding the non-intrusive sound source confirmed its applicability in acoustic shielding experiments. Because of the very short duration of the produced pressure pulse $(\approx 50 \mu \mathrm{s})$ any acoustic reflection can be identified and rejected during data post-processing. This consequently extend the domain of application of the technique to traditional aerodynamic wind tunnels with no acoustic treatment.

\section{References}

${ }^{1}$ Pott-Pollenske, M., Dobrzynski, W., Buchholz, H., Guérin, S., Saueressig, G., and Finke, U., "Airframe noise characteristics from flyover measurements and predictions," AIAA Paper 2006-256\%., , No. 2567, 2006.

${ }^{2}$ Bertsch, L., Dobrzynski, W., and Guérin, S., "Tool development for low-noise aircraft design," 14 th AIAA/CEAS Aeroacoustics Conference, 5-7 May, Vancouver, Canada 2008.

${ }^{3}$ Stone, J., Groesbeck, D., and Zola, C., "Conventional profile coaxial jet noise prediction," AIAA Journal, Vol. 21, No. 1, 1983, pp. 336-342.

${ }^{4}$ Heidmann, M., "Interim prediction method for fan and compressor source noise," NASA Technical Report, , No. TM-X-71763, 1979.

${ }^{5}$ Delfs, J., Boden, F., and Buchholz, H., "Entwicklung einer neuartigen Laserschallquelle," Proceedings DAGA, Braunschweig, Braunschweig, 2006.

${ }^{6}$ Kirkup, S. M., "BEMHELM: BEM for Helmholtz Problems," http://www.boundary-elementmethod.com/helmholtz/manual/index.htm, 1998.

${ }^{7}$ Benthien, W. and Schenck, A., "Non-existence and Non-uniqueness Problems associated with Integral Equation Methods in Acoustics," Computers \& Structures, Vol. 65, No. 3, 1997, pp. 295-305.

${ }^{8}$ Esward, T. J., Lees, K., Sayers, D., and Wright, L., "Testing Continuous Modelling Software: Three Case Studies," Report CMSC 42/04, NPL, 2004.

${ }^{9}$ Burton, A. J. and Miller, G. F., "The Application of Integral Equation Methods to the Numerical Solution of some Exterior Boundary Value Problems," Proceedings of the Royal Society, London, Vol. A 323, 1971, pp. 201-210.

${ }^{10}$ Radziemski, L. and Cremers, D., Laser induced plasmas and applications, Marcel Dekker Inc., New-York, June 1989.

${ }^{11}$ Boden, F. and Delfs, J., "Development of a laser-based sound source," Inter-Noise, Honolulu, Hawaii; USA, 3-6 December 2006.

${ }^{12}$ Boden, F., "Grundlagenuntersuchungen zu einer neuartigen Laserschallquelle," Tech. Rep. IB 124-2007/5, DLR, Braunschweig, Institut für Aerodynamik und Strömungstechnik, 2007. 